\title{
Classification of Coronary Artery Disease Using Different Machine Learning Algorithms
}

\author{
Bahar Nazlı \\ Department of Biomedical Engineering, Kastamonu University, Kastamonu, Turkey \\ E-mail: bnazli@kastamonu.edu.tr \\ Yasemin Gültepe \\ Department of Computer Engineering, Kastamonu University, Kastamonu, Turkey \\ Hayriye Altural \\ Department of Biomedical Engineering, Kastamonu University, Kastamonu, Turkey
}

Received:13 April 2020; Accepted: 13 May 2020; Published: 08 August 2020

\begin{abstract}
Coronary Artery Disease (CAD) takes place in the category of fatal diseases resulting in death in our country and around the world. Each year about 340 thousand patients lost their lives due to CAD in Turkey. Early diagnosis is essential to reduce risk and prolong lifetime of these patients for diseases that require long-term treatment having death risk like CAD. For this reason, classification of CAD by using medical data processing and machine learning algorithms are important in order to develop assistive or expert systems for physicians. In this study, five different machine learning algorithms were applied to estimate whether patients in the Z-Alizadeh Sani data set extracted from the UCI machine learning pool are CAD. Accuracy, precision, recall, specificity and F1 score were compared as classification performance indicators to evaluate decision tree, random forest (RF), support vector machines (SVM), nearest neighborhood (k-NN) and multi-layer sensor (MLP) methods. According to the evaluation results, the MLP method gave high classification accuracy with $90 \%$. It also appears that RF performs relatively better than other metrics. This results, show that these classification algorithms can be use for helping healthcare systems.
\end{abstract}

Index Terms: Coronary artery disease, classification, machine learning, MLP.

\section{Introduction}

Coronary artery disease (CAD), as in the world, in our country it is one of the leading reasons of death and morbidity. Early and accurate detection of CAD has critical importance in its management [1]. Clinical decision making processes are often based on physicians' intuition and experience. This approach can negatively affect the quality of healthcare provided to patients by increasing unwanted prejudices, clinical errors and medical costs.

Machine learning methods show high performance in the diagnosis of diseases with objective algorithms for analysis of high-dimensional and various biomedical data. It is not possible to process and analyze huge amounts of data manually. However, it is often possible to make predictions and inferences for the future using historical data. Machine learning methods are used to make these inferences [2].

Therefore, researchers are working on alternative methods such as machine learning (ML) in the diagnosis of CAD. With the use of ML methods in clinical fields, all available variables for patients can be easily interpreted and evaluated, and thus the diagnostic accuracy of each step can be increased [3, 4]. In recent years, the use of ML has significantly increased in the biomedical data analysis, the diagnosis and detection of diseases [5].

For this purpose, this study aims to increase the predictive accuracy rate of coronary artery disease by using machine learning classification algorithms. In this study, the classification process with ML algorithm is carried out in three stages: data preprocessing, classification model training and estimation evaluation [6]. Implementation of ML algorithms requires data to be in a mathematically appropriate with data preprocessing. Data preprocessing techniques consist of data reduction, data projection and completion of missing data [7]. ML is defined as computer programming to make descriptive or predictive inferences using sample data or benefiting past experience with inductive methods [8]. So as to evaluate the performance of ML models, the certain metrics accepted as standard are applied. 
The researchers do experiments on common use datasets to test effectiveness of methods they developed. In this study, Z-Alizadeh Sani datasets in the University of California at Irvine (UCI) ML repository were used [9,10]. In this study, after the data in the raw data set were normalized by minimum-maximum normalization techniques, classification was done by machine learning classification methods. Different models have been created using decision tree (DT), random forest (RF), support vector machines (SVM), k-nearest neighborhood (k-NN), multi-layer sensor (MLP) algorithms. Classification performances of these models are compared.

In section 2, a literature research summarizing the studies in the field of CAD related machine learning is given. In section 3, the materials and methods of this study are mentioned and given in detail. In section 4, which is the application phase of the study, experimental results are presented. In section 5, there are suggestions for evaluating the results obtained in the study and to guide future scientific studies.

\section{Previous Studies}

In this section, a brief description of classical parallel adders used as sub-adders constituting a hybrid adder is introduced. This description can be found in literature. However, for the sake of completeness we briefly explain the concept of the different classical parallel adders associated with appropriate references.

There are different studies about estimation of CAD in the literature. Alizadehsania et al. used computational intelligence method for detection which arteries are stenosed. They achieved the accuracy rates of $86.14 \%, 83.17 \%$ and 83.50\% for the diagnosis of left anterior decending artery, left circumflex artery and rigth coronary artery stenosis using the Alizadeh Sani dataset, respectively [10]. Dolatabadi et al. predicted the presence of heart disease by applying a classification method on the Long-Term ST dataset of ECG records of 80 patients with CAD and the Normal Sinus Rythm RR Interval dataset of Holter records of 56 healthy people as a control group. Using the SVM method, the dataset was classified as patient and patient, and the highest parameter values such as $99.2 \%$ accuracy, 98.43\% sensitivity and $100 \%$ specificity were achieved [11].

Arabasadi et al. used Hybrid Neural Network and Genetic Algorithm together for the detection of CAD cases. In their study, the performance of the neural network increased about $10 \%$ in the Genetic Algorithm application in order to suggest better weights for neural networks. Feature selection was made using SVM weights in the Z-Alizadeh Sani dataset. These methods with a success rate of $93.85 \%$ have $97 \%$ sensitivity and $92 \%$ selectivity values [12].

Giri et al. used SVM, Gaussian Mix Model (GMM), Probabilistic Neural Network and k-NN methods as the learning algorithms to determine the abnormalities in ECG signals recorded with the BIOPAC system. It had been proven that the accuracy, sensitivity and selectivity values gave the best results with the highest values of $96 \%$ accuracy, 100\% sensitivity and $93.7 \%$ selectivity when Independent Component Analysis feature extraction methods were combined with the GMM classifiers [13]. In another study conducted by Alizadehsani et al., the most appropriate algorithm was determined to develop a classification model in the detection of CAD cases by comparing Naïve Bayes, Sequential Minimal Optimization (SMO), Bagging, Neural Network algorithms on the Z-Alizadeh Sani dataset. The highest accuracy value was obtained as $94.08 \%$ with the SMO algorithm used with the feature selection on extracted features and feature extraction [14].

Swarnava et al. applied a learning method to improve a classification model for the diagnosis of CAD by using MIMIC-II and local hospital datasets. In their study, feature extraction was done from ECG signal by using Wavelet Transform and the SVM classifier algorithm was used in the classification phase. For the MIMIC-II dataset, 89\% accuracy, $86 \%$ sensitivity and $90 \%$ selectivity parameters were obtained. In the experimental study using the other dataset obtained from the local hospital, 93\% accuracy, 92\% sensitivity, and 94\% selectivity parameters were obtained [15]. In the study conducted by Bektaş, data mining algorithms applied to classification of CAD. For this purpose, three dataset filling methods were used: k-means, MLP and Self Organizing Map. The 90\% sensitivity and 18\% specificity parameters with their highest values were obtained for a public hospital dataset by using MLP method. A mixed classification process was performed in two different datasets by using linear regression (LR) and SVM methods. In their conclusion, the effective algorithm, solving problems by training the entire unbalanced dataset at once with this classification process was improved [16].

In the thesis study conducted by Cihan, the risk of CAD was analyzed by using the RF algorithm on Cleveland and Hungary heart disease datasets extracted from the UCI dataset collection. In the classification model applied in the study, an accuracy of $86.13 \%$ was achieved on the Celeveland dataset. In addition, the data set consisting of 596 patient records obtained by combining Hungary-Cleveland datasets achieved 80\% accuracy. Chest pain type and exerciseinduced ST segment depression variables in both datasets were identified as the most important variables for the classification [17].

\section{Material and Method}

In this study, Python version 3.7 platform, which is easy to learn, easy to read was used and has many ready data science libraries. This platform was preferred because it contains the libraries such as "Numpy", "Pandas", "ScikitLearn" and "Keras" which are the most basic and widely used for data science studies. 


\subsection{Dataset}

In this study, the Z-Alizadeh Sani dataset extracted from UCI ML repository was used as the dataset [9]. The dataset consists of 303 patient records, containing 54 features for each. The features were examined in four categories as demographic, symptom/examination, ECG/laboratory and eco features. Each patient was examined in two groups, with or without coronary artery disease. A patient, diameter narrow of artery is less than $50 \%$, is labeled as Normal, otherwise she/he as CAD. There are 97 healthy and 216 CAD subjects in this dataset.

\subsection{Data analysis}

Before proceeding to the classification stage, it is necessary to prepare the dataset by pre-processing applications such as normalization and cross-validation on the dataset to obtain higher accuracy [18]. In this study, all values which appear as "objects" in the dataset were first converted into numerical values and made ready for processing. The normalization process is performed to the raw data and has an impact on the preparation of the appropriate dataset for training. Several techniques can be used in normalization processes. There are many types of data normalization in the literature. These can be listed as rules such as minimum-maximum, Median, Sigmoid and Z-Score rules etc [19]. In order to observe the effect of normalization techniques on machine learning performance, the minimum-maximum normalization technique was used to normalize the data in this study. Using normalized data will produce more accurate results.

Before proceeding to the classification phase, K-fold cross-validation method, one of the training-test methods, was used for the data set that should be separated as training and test. Selecting the most appropriate k value can provide more successful results. The classifiers given in Table 1 on the Z-Alizadeh Sani dataset were tried sequentially with parameters $\mathrm{k}$ from 1 to 10 and accuracy ratios were obtained by cross validation. High classification accuracy values were obtained by using 10 -fold cross validation method. Thus, it was decided to use 10 -fold cross-validation method.

Table 1. K-fold cross validation values

\begin{tabular}{cc}
\hline Methods & K-fold Value \\
\hline DT & 8 \\
RF & 10 \\
SVM & 8 \\
k-NN & 5 \\
MLP & 5 \\
\hline
\end{tabular}

\subsection{Algorithm selection}

The aim of this study is to apply different classification algorithms based on machine learning to predict CAD using Decision Trees, Random Forest, Support Vector Machines, k-Nearest Neighborhood, Multilayer Perceptron algorithms on the data set obtained after applying the minimum-maximum normalization technique on the raw data set. The algorithms are described as follows, respectively:

Decision Trees: It is expressed as class tags at the level of the leaves of the tree and operations on features with branches going to these leaves and spreading from the beginning by creating a tree structure [20]. The algorithm is simple in terms of understanding and interpretation. It can be used for both numerical and class processing.

Random Forest: Low depth of the decision tree causes the classification not to be realized, also its high depth makes the classification difficult. Tree depth is reduced by increasing the number of trees in RF [21]. It is possible to determine correct classifications among trees in RF by bagging. Bagging is done with calculations called voting in classification problems. High performance of a random decision tree is possible by using appropriate number of trees and correct voting between trees.

Support Vector Machines: It is a nonparametric classification algorithm based on statistical learning theory [22]. SVMs have been developed for dual classifications. The working principle of SVM is based on estimating the most appropriate decision function that can separate two classes from each other, in other words, defining the hyper-plane that can separate two classes in the most appropriate way.

k-Nearest Neighborhood: In this algorithm, the classification process is made by considering relationships between data [23]. This system works on the coordinate plane with the linear decomposition method. Object to be classified in original k-NN algorithm is assigned to the class included the majority according to the nearest $\mathrm{k}$ neighbors of this object [24].

Multilayer Perceptron: Artificial neural networks are learning algorithms created by modeling human neural cells [25]. They consist of an input layer from which information is entered, one or more hidden layers and an output layer. In MLPs, there are transitions called forward propagation and backward propagation. In forward propagation stage, the output and error value of the network are calculated. In the back propagation stage, link weight values between the layers are updated to minimize the calculated error value [26]. 


\subsection{Evaluation criteria}

All experiments in this study were done using Python program on HP desktop computer which has Windows 10 Education 64 bit operating system with $3.20 \mathrm{GHz}$ processor speed and $4 \mathrm{~GB}$ of RAM.

The success of the models created using different machine learning algorithms directly depends on the number of true estimates. Accuracy of classification is calculated to true estimation of the models that is created or choosen for classification problems. However, that accuracy not demonstrated sufficient information by alone. Accuracy metrics calculated form confusion matrices are used for obtained good and adequate results.

In this study, the confusion matrices obtained from the algorithm results were calculated one by one with the general valid evaluation criteria used in medicine. Thanks to these criteria, the algorithms that can be used most effectively in the diagnosis and diagnosis of CAD disease can be determined.

Confusion matrix: The confusion matrix is a table containing values of comparing actual datas and results of model. This matrix is often used by obtain open and clear estimated results of a classifier [27]. Confusion matrix contains the following terms;

True Positive (TP): The number of true predictions that are positive for a model. In this study, it gives the number of actual patients classified as patients.

True Negative (TN): The number of false predictions that are true for a model. In this study, it gives the number of people who are not actually classified as patients.

False Positive (FP): The number of false predictions that are positive for a model. In this study, it gives the number of people who are classified as patients but are not patients.

False Negative (FN): The number of false predictions that are negative for a model. In this study, it gives the number of actually patients who are not classified as patients.

Confusion matrix give the neccessery information about classification performance of model. Nevertheless, since confusion matrix does not give a single result to compare the performance of different models, the results must be converted a single value. This can be done using performance criteria such as accuracy, precision, sensitivity, F1-score, as follows [28]:

Accuracy: It measures how much of a model's estimates are correct. In this study, it is the ratio of people classified as patients to the total number of people. Accuracy is explained as follows:

$$
\text { Accuracy }=\frac{\mathrm{TP}+\mathrm{TN}}{\mathrm{TP}+\mathrm{TN}+\mathrm{FP}+\mathrm{FN}}
$$

Recall: It gives the ratio of items classified as positive to items that are actually positive. In this study, it is the number that gives the ratio of patients classified as patients to the number of patients in real. Recall is given by the relation:

$$
\text { Recall }=\frac{T P}{T P+T N}
$$

Specificity: It gives the ratio of items not classified as positive to items that are actually positive. In this study, it is the number that gives the ratio of non-patients to non-patients who are not actually classified as patients. Specificity is defined as follows:

$$
\text { Specificity }=\frac{F P}{F P+F N}
$$

Precision: It is defined as number of true classified of positive samples divide by number of total positive sample. Prescision shows the effectiveness of the model in predicting the positive class tag from inputs. In this study, it is the ratio of people who are actually patient and classified correctly to people who are classified as patients. Precision is calculated as follows:

$$
\text { Precision }=\frac{T P}{T P+F P}
$$

F1-Score: It is the harmonic average of precision and sensitivity performance evaluation criteria. It allows to evaluate both criteria together. It is more useful than considering the accuracy value for the F1 score, especially when there is an unequal class distribution. In the most real-life classification problems, there is an unbalanced class distribution and therefore the F1-score is a better criterion to evaluate the model. F1-score is given in the following equation:

$$
F 1-\text { Score }=\frac{2 * T P}{2 * T P+F P+F N}
$$

In addition, Mean Absolute Error (MAE), Root Mean Square Error (RMSE) and Mean Square Error (MSE) are among the main performance measurement criteria used in the evaluation of model performance in Machine Learning methods. MSE, RMSE and MAE are calculated as given following equations 6, 7 and 8 respectively.

$$
\operatorname{MSE}=\frac{1}{n} \sum_{i=1}^{n} e_{i}^{2}
$$




$$
\begin{aligned}
\text { RMSE } & =\sqrt{\frac{1}{n} \sum_{i=1}^{n} e_{i}^{2}} \\
\text { MAE } & =\frac{1}{n} \sum_{i=1}^{n}\left|e_{i}\right|
\end{aligned}
$$

In these formulas 'e' represent to error. MSE, RMSE and MAE are measures that lower values indicate better performance because each of them is a measure of error [29]. For example, if RMSE is equal to zero, it can be said to indicate a better performance result. RMSE, MAE and MSE criteria were used to measure the model prediction success of the five methods discussed in this study. RMSE, MAE and MSE values close to 0 indicate that very important mistakes were not made.

\section{Results and Discussion}

In this study, Decision Trees, Random Forest, Support Vector Machines, k-Nearest Neighborhood and Multilayer Perceptron methods were used. Parameters such as accuracy, sensitivity, specificity, precision, F1-score were used to measure classification algorithm performances. When all the results obtained are analyzed, the best performing classifiers in CAD detection are summarized in Table 2. According to Table 2, the MLP classifier was successful in the classification of CAD with the highest accuracy value of $90 \%$. These results show that the MLP Algorithm performs better for this problem than other classification algorithms. According to the graph shown in Figure 1, MLP algorithm gives the best accuracy result, followed by SVM, DT, RF and k-NN algorithm, respectively.

Table 2. Cross-validation test results for ML algorithms

\begin{tabular}{lccccc}
\hline Criteria & $\begin{array}{c}\text { DT } \\
(\%)\end{array}$ & $\begin{array}{c}\text { RF } \\
(\%)\end{array}$ & $\begin{array}{c}\text { SVM } \\
(\%)\end{array}$ & $\begin{array}{c}\text { k-NN } \\
(\%)\end{array}$ & $\begin{array}{c}\text { MLP } \\
(\%)\end{array}$ \\
\hline Accuracy & 81.08 & 80.0 & 83.78 & 80.00 & 90.00 \\
Recall & 75.0 & 64.71 & 75.0 & 75.86 & 82.76 \\
Specificity & 88.24 & 100 & 94.12 & 83.87 & 96.77 \\
Precision & 88.24 & 100 & 93.75 & 81.48 & 96.00 \\
F1-Score & 81.08 & 78.57 & 83.33 & 78.56 & 88.88 \\
\hline
\end{tabular}

The cross-validation test results calculated as mathematical values in Table 2 are shown as bar graph in Figure 1. As shown in Fig. 1, the highest specificity and precision rates belong to RF method.

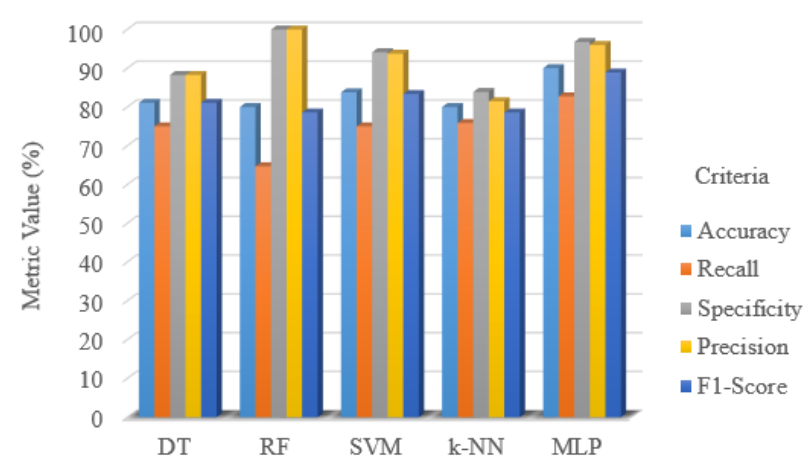

Fig. 1. Cross-validation test results for ML algorithms

Performance results of MAE, RMSE and MSE values according to five different ML algorithms used in the study were given in Table 3. In the literature studies we investigated, no study using error analysis to compare classification performances in CAD classification studies has been found. Diffirent from other studies, in order to make a more detailed comparision to each other algorithms, MSE, RMSE and MAE error analysis were added for the performance evaluation of the study. This is seen as the most important stage that ensures that our study has original value. As given in Table 3, considering that lower MAE, RMSE and MSE values indicate better performance, it can be said that the performance results of MLP indicate relatively lower values in terms of MAE (0.1), RMSE (0.3162) and MSE (0.1), in other words, these performance results are better. This results support that other performance metrices. When the lowest value indicates the best error rate, the highest value corresponds to the highest error rate. It is seen that the most obvious evaluation criterion for determining the difference between the models is RMSE calculation. 
Table 3. Comparison of the performance of ML algorithms in the way of MAE, RMSE and MSE

\begin{tabular}{lccccc}
\hline Metrics & DT & RF & SVM & k-NN & MLP \\
\hline MAE & 0.1892 & 0.2 & 0.1622 & 0.2 & 0.1 \\
RMSE & 0.435 & 0.4472 & 0.4027 & 0.4472 & 0.3162 \\
MSE & 0.1892 & 0.2 & 0.1622 & 0.2 & 0.1 \\
\hline
\end{tabular}

\section{Conclusion}

Machine learning is widely used in medicine as well as in many different fields and plays a supportive system in the diagnosis of diseases. In the study, 54 features of 313 people, 97 healthy and 216 CAD patients, were used, and CAD disease was detected with these features. The aim of the study is to find the best performing classifier among five different machine learning classifiers in CAD disease detection. In the experiment conducted, the training and test performance of the system was measured with cross verification. For cross validation, $k=10$ is taken. The results of the study are summarized in Table 2 and it has been shown that the MLP classifier algorithm showed the highest performance (accuracy $=90 \%$, recall $=82.76 \%$, specificity $=96.77 \%$, precision $=96.00 \%$ and F1-score $=88.88 \%$ ) in CAD detection. The results of the MLP classifier from the error rate calculations were calculated as MAE, RMSE, MSE as $0.1,0.3162$ and 0.1 , respectively. With the machine learning techniques, computers gain the ability to make autonomous decisions by learning the information provided by human experts. In this way, support to experts plays a system role, and as learning data increase and diversify, they can produce more successful results.

\section{References}

[1] C. Padmavathi, V. S. Veenadev, "An Automated Detection of CAD Using the Method of Signal Decomposition and Non Linear Entropy Using Heart Signals,” International Journal of Image, Graphics and Signal Processing, 2019, 11(2), pp. 30-39.

[2] P. Harrington, "Machine Learning in Action”, 1st Edition, Manning Publications Shelter Island, NY, ISBN: 978-1-61729-018-3.

[3] J. Soni, U. Ansari, D. Sharma, S. Soni, "Predictive data mining for medical diagnosis: An overview of heart disease prediction”, International Journal of Computer Applications, 2011, 17 (8), pp. 43-48.

[4] M. L. Sharan, K. B. Sathees, "Analysis of cardiovascular heart disease prediction using data mining techniques”, International Journal of Modern Computer Science, 2016, 4 (1), pp. 55-58.

[5] K. R. Foster, R. Koprowski, J. D. Skufca, "Machine learning, medical diagnosis and biomedical engineering researchcommentary”, Biomedical Engineering Online, 2014, 13 (1), p. 94.

[6] K. N. A. Halim, A. S. M. Jaya, A. Firdaus, "Data pre-processing algorithm for neural network binary classification model in bank tele-Marketing”, International Journal of Innovative Technology and Exploring Engineering, 2020, 9 (3), pp. 272-277.

[7] J. Huang, Y.-F. Li, M. Xie, “An empirical analysis of data preprocessing for machine learning-based software cost estimation”, Information and Software Technology, 2015, (67), pp. 108-127.

[8] J. Vamathevan, D. Clark, P. Czodrowski, I. Dunham, E. ferran, G. Lee, B. Li, A. Madabhushi, P. Shah, M. Spitzer, S. Zhao, "Applications of machine learning in drug discovery and development”, Nature Reviews Drug Discovery, 2019,18 (6), pp. 463477.

[9] UCI Machine Learning Repository, Z-Alizadeh Sani Data Set, 2017, [Online]. Available: https://archive.ics.uci.edu/ml/datasets/Z-Alizadeh+Sani.

[10] R. Alizadehsani et al., "Coronary artery disease detection using computational intelligence methods”, Knowledge-Based Systems, 2016, (109), pp. 187-197.

[11] A. D. Dolatabadi, S. E. Z. Khadem, B. M. Asl, “Automated diagnosis of coronary artery artery disease (CAD) patients using optimized SVM”, Computer Methods Programs in Biomedicine, 2017, (138), pp.117-126.

[12] Z. Arabasadi, R. Alizadehsani, M. Roshanzamir, H. Moosaei, A. A. Yarfard, "Computer aided decision making for heart disease detection using hybrid neural network-genetic algorithm”, Computer Methods Programs in Biomedicine, 2017, (141), pp. 19-26.

[13] D. Giri, U. R. Acharya, R. J. Martis, S. V. Sree, T.-C. Lim, T. Ahamed VI, J. S. Suri, “Automated diagnosis of coronary artery disease affected patients using LDA, PCA, ICA and Discrete Wavelet Transform”, Knowledge-Based Systems, 2013, (37), pp. 274-282.

[14] R. Alizadehsani et al., “A data mining approach for diagnosis for coronary artery disease”, Computer Methods and Programs in Biomedicine, 2013, 111 (1), pp. 52-61.

[15] D. Swarnava, B. Swagata, P. Arpan, A. Mukherjee, G. Utpal, M. Kayapanda, “CAD patient classification using MIMIC-II”, 2017, Institute for Computer Sciences, Social Informatics and Telecommunications Engineering, 2017, (181), pp. 370-375.

[16] J. Bektaş, "Use of mining techniques to determine presence of coronary artery disease and deriving a risk score by employing risk factors”, Doctoral Thesis, 2017, Çukurova University Institute of Natural and Applied Sciences, p. 101.

[17] Ş. Cihan, "Analyzing the risk of coronary artery disease using machine learning, Master Thesis, 2018, Kırıkale University, Graduate of Computer Engineering, p. 111.

[18] S. G. K. Patro, K. K. Sahu, “Normalization: A preprocessing stage”, Computer Science, Published in ArXiv, 2015.

[19] T. Jayalaksmi, A. Santhakumaran, "Statistical normalization and back propagation for classification", International journal of computer theory and engineering, 2011, 3 (1), pp. 1793-8201. 
[20] Y. Y. Song, Y. Lu, “Decision tree methods: Applications for classification and prediction”, Shanghai Archives of Psychiatry, 2015, 27 (2), pp. 130-135.

[21] M. Belgeiu, L. Dragut, "Random forest in remote sensing: A review of applications and future directions", ISPRS Journal of Photogrammetry and Remote Sensing, 2016, (114), pp. 24-31.

[22] V. N. Vapnik, “The nature of statistical learning theory”, New York: Springer-Verlang, 1995.

[23] V. Vijayalakshmi, K. Venkatachalapathy, "Comparison of Predicting Student's Performance using Machine Learning Algorithms”, I.J. Intelligent Systems and Applications, 2019, 11(12), pp. 34-45.

[24] Y. Wu, K. Ianakiev, V. Govindaraju, “Improved k-nearest neighbor classification”, Pattern Recognition, 2002, (35), pp. 23112318.

[25] E. O. O. Olaniyi, O. K. Oyedotun, K. Adnan “Heart diseases diagnosis using neural networks arbitration”, I.J. Intelligent Systems and Applications, 2015, 12, pp. 75-82.

[26] S. Shanmuganathan, "Artificial neural network modelling: An introduction”, Sentiment Analysis on Morphologically Rich Languages: An Artificial Neural Network (ANN) Approach, 2016, pp. 1-14.

[27] M. Sokolova, “A systematic analysis of performance measures for classification tasks”, Information Processing \& Management, 2009, 45(4), pp. 427-437.

[28] M. Sokolova, G. Lapalme, “A systematic analysis of performance measures for classification tasks”, Information Processing and Management, 2009, 45, pp: 427-437.

[29] W. Wang, Z. Xu, “A heuristic training for support vector regression”, Neurocomputing, 2004, 61, pp. 259-275.

\section{Authors' Profiles}

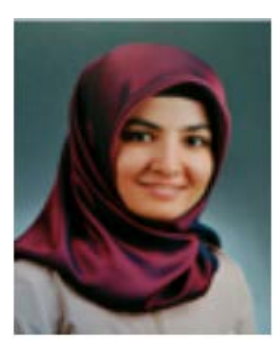

Bahar Nazlı is currently working as Research Assistant of Biomedical Engineering Department at Kastamonu University, Kastamoun/Turkey. She has completed her B.Sc degree in Biomedical Engineering Department from Erciyes University. She continues M.Sc. degree in Biomedical Engineering Department at Kastamonu University. Her research is in the field of signal processing and machine learning.

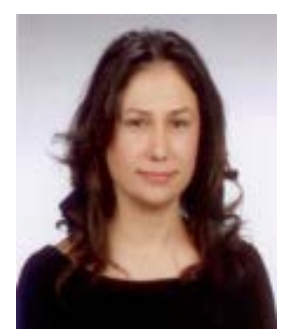

Yasemin Gültepe is currently working as Assistant Professor of Computer Engineering and Head of Computer Engineering Department at Kastamonu University. She has completed her B.Sc. and M.Sc. degree in Computer Engineering Department from Çanakkale Onsekiz Mart University, Çanakkale/Turkey. She has completed her doctor of philosophy in Institute of Sciences at Ege University, Izmir/Turkey. Her research is in the field of semantic web, machine learning, information management.

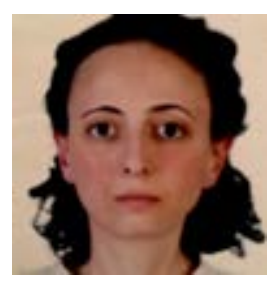

Hayriye Altural received her B.Sc. in electronic engineering at Erciyes University and her Ph.D. in optoelectronics at the same university. After post-doctoral positions at Boston University and Iowa State University, Dr. Altural joined the faculty at the Kastamonu University in 2016. She is now assistant professor in the Biomedical Engineering Department. Her research interests focus on design of biomedical devices and sensors.

How to cite this paper: Bahar Nazlı, Yasemin Gültepe, Hayriye Altural. " Classification of Coronary Artery Disease Using Different Machine Learning Algorithms ", International Journal of Education and Management Engineering (IJEME), Vol.10, No.4, pp.1-7, 2020. DOI: 10.5815/ijeme.2020.04.01 